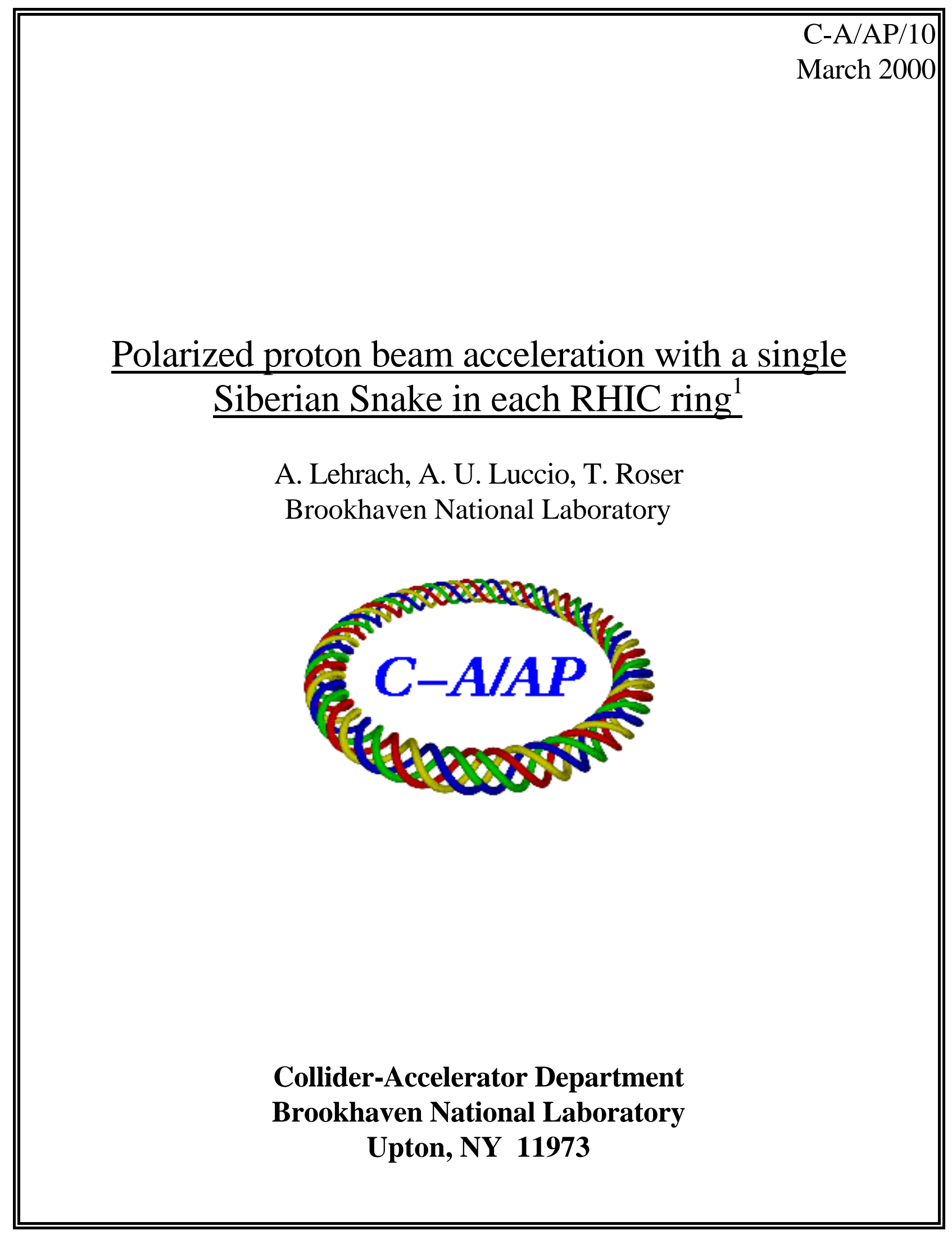




\title{
Polarized proton beam acceleration with a single Siberian Snake in each RHIC ring ${ }^{1}$
}

\author{
A. Lehrach, A.U. Luccio, T. Roser
}

Brookhaven National Laboratory, P.O. Box 5000, Upton, New York 11973, USA

\begin{abstract}
This paper describes a method to accelerate polarized proton beams with a single snake in each RHIC ring to half maximum energy and deliver longitudinal beam polarization to all interaction points.
\end{abstract}

\section{Introduction}

Siberian Snakes are used to overcome depolarizing resonances in circular accelerators by forcing the spin tune to half integer, independent of beam energy. This concept has been proposed by Ya.S. Derbenev and A.M. Kondratenko in 1974 [1]. A single Siberian Snake rotates the spin by $180^{\circ}$ at each turn around a horizontal axis (snake axis). Two snakes in each ring are sufficient to preserve the beam polarization up to the maximum energy of RHIC [2]. The direction of the spin, that is invariant after one turn is called the stable spin direction. The beam polarization is only preserved in this direction. The stable spin direction in both RHIC rings is vertical in a two snake scheme. Spin rotators provide longitudinal beam polarization at the interaction points of the STAR and PHENIX experiment.

If only a single snake in each ring is used, the stable spin direction is in the horizontal plane. By adjusting the snake axis in the horizontal plane, it is also possible to deliver longitudinal beam polarization at the interaction points of RHIC, which will not be equipped with spin rotators, like the one at the pp2pp experiment.

\section{Spin motion with a single snake in RHIC}

The orientation of the snake axis ${ }^{2}$ in the horizontal plane is defined by the angle $\phi$ with respect to the beam direction (longitudinal direction). In a single snake scheme the stable spin direction is along the snake axis at the so called symmetry point of the snake, located at the opposite side of the snake in the ring. At any other point in the accelerator the stable spin direction is somewhere in the horizontal plane, depending on the beam energy. To simulate the behavior of the spin during acceleration with a single snake, the spin tracking program SPINK was used [3].

\footnotetext{
${ }^{1}$ Work performed under the auspices of the U.S. Department of Energy.

${ }^{2}$ The nominal setting of the snake axis in RHIC is $\phi=+45^{\circ}$ and $-45^{\circ}$ for the two snakes in each ring.
} 
In the presented simulation the snake next to 10 o'clock in the blue ring ${ }^{3}$ was operated with a snake axis of $\phi=-45^{\circ}$ during acceleration. A beam with a randomly distributed normalized transversal emittance of $20 \pi \mathrm{mm}$ mrad was chosen. The RHIC lattice has a random sample of quadrupole misalignments with a rms spread of $\pm 0.5 \mathrm{~mm}$ and beam position monitor misalignments of $\pm 0.2 \mathrm{~mm}$. The orbit correction scheme MICADO was applied to correct the vertical closed orbit [4].

\subsection{Spin preparation after injection}

The polarized proton beam is injected into RHIC with vertical polarization. To preserve the beam polarization using a single snake in each RHIC ring, the spin vectors have to be along the stable spin direction. This can be achieved, if the snake is adiabatically turned on after injection and is ramped until the spin vectors are in the horizontal plane. During this process, the spin tune changes from $\nu_{s}=\gamma G$ (spin tune without snake) to the nearest half integer tune, and depolarizing resonances can be crossed. To avoid crossing these resonances, the snake has to be turned on close to a half integer spin tune. Simulations starting at different injection energies are shown in Fig. 1. If the snake is turned on at an
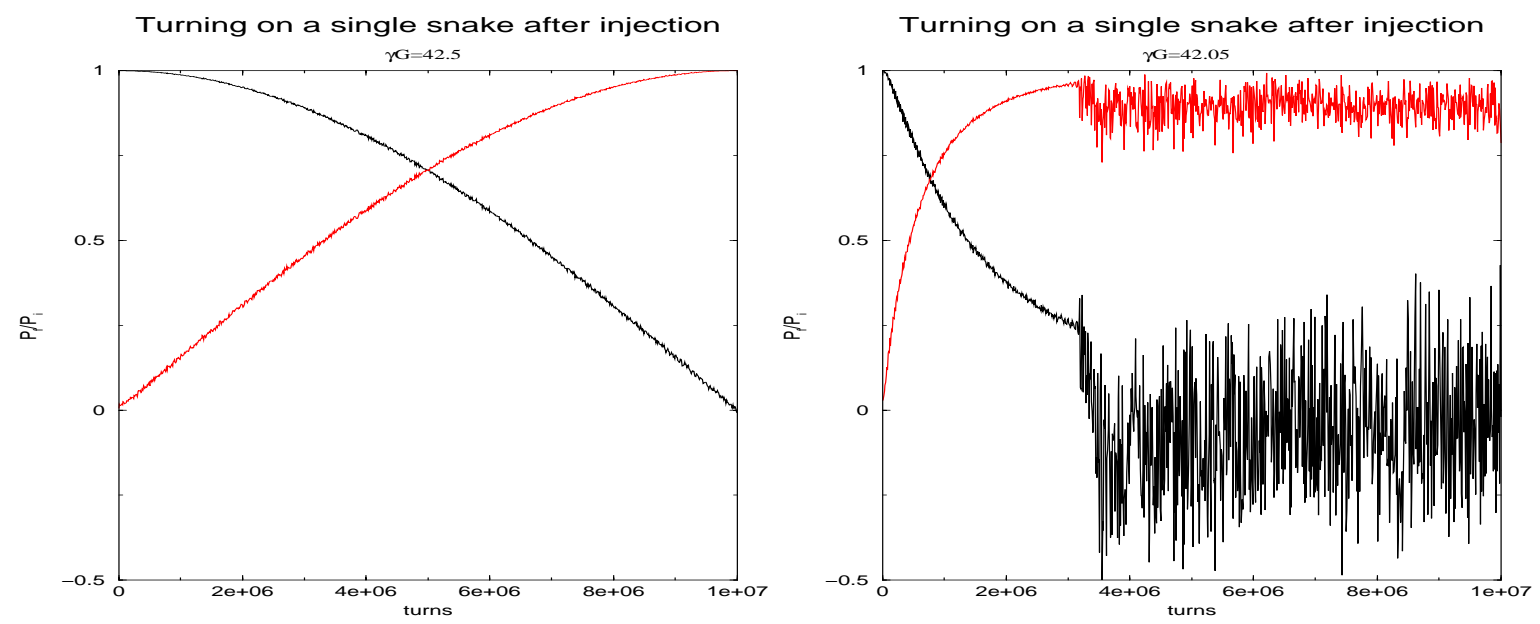

Figure 1: Beam polarization during transferring the spin from the vertical direction into the horizontal plane. The vertical and horizontal ratio of polarization $P_{f} / P_{i}$ is plotted. The snake is turned on at two different injection energies corresponding to $\gamma G=42.5$ and 42.05 . It is ramped linearly in 10000000 turns (about $128 \mathrm{sec}$ ) to the norminal spin rotation angle of $180^{\circ}$.

energy corresponding to half integer spin tune, no depolarzing resonances are crossed. The polarization is preserved during spin preparation. For injection energies corresponding to spin tunes which are close to an integer, depolarizing resonances are crossed, which leads to polarization losses.

\subsection{Acceleration with a single snake}

Due to the chosen orientation of the snake axis, the stable spin direction at the symmetry point is also $-45^{\circ}$ with respect to longitudinal direction. The spin tracking results using an acceleration rate of $0.84 \cdot 10^{-5} \mathrm{GeV}$ per turn for a one and two snake scheme is presented in Fig. 2. The simulation shows, that it is possible to accelerate a polarized proton beam with a one snake scheme above $100 \mathrm{GeV}(\gamma G=191)$ without major polarization losses.

\footnotetext{
${ }^{3}$ Particles are circulation clockwise in the blue ring and anti-clockwise in the yellow ring.
} 


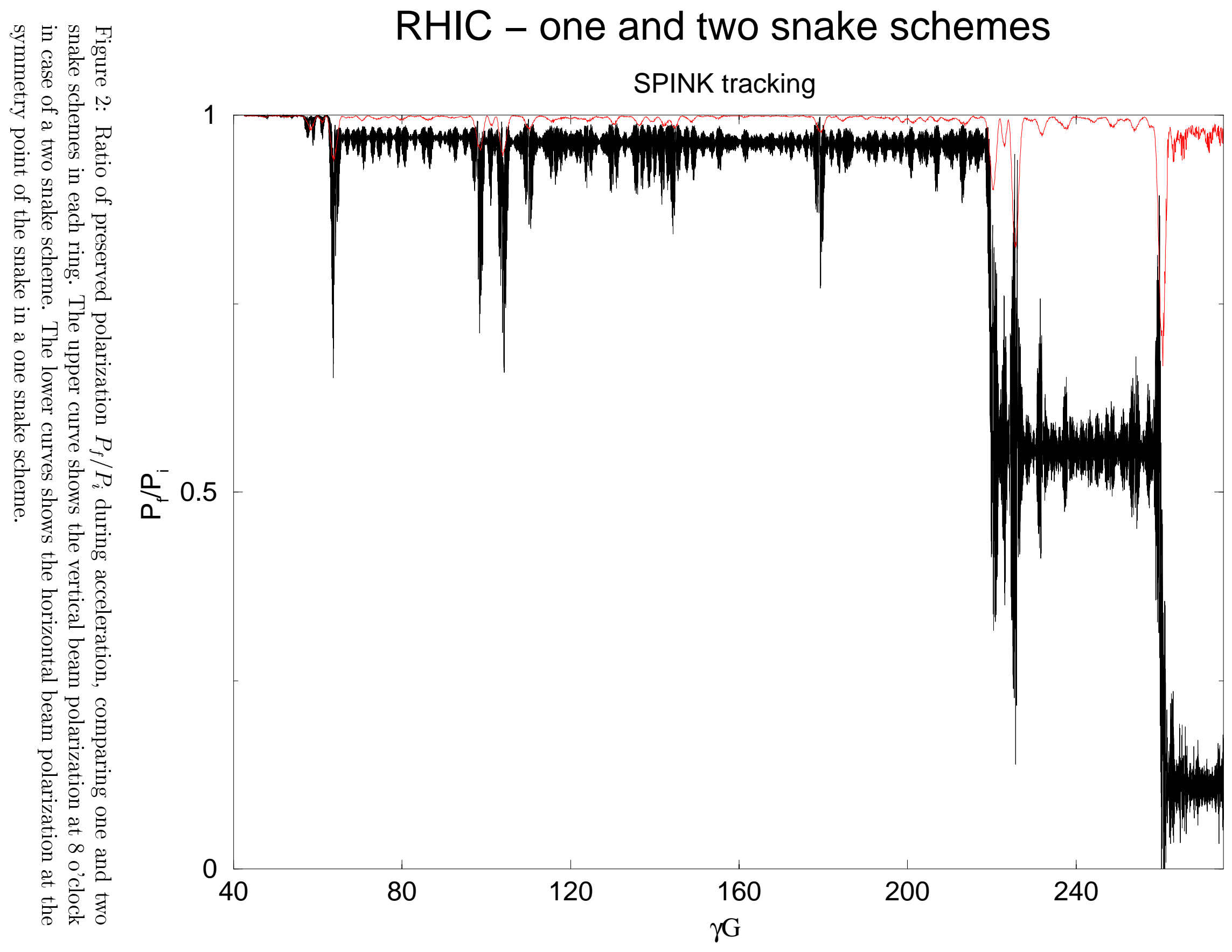


The ratio of preserved polarization is higher than 95\%. First large polarization losses occur at about $115 \mathrm{GeV}(\gamma G=220)$. The ratio of preserved polarization at energies above $140 \mathrm{GeV}(\gamma G=260)$ is below $15 \%$. Compared to the two snake scheme, the spread of the spin vectors is also bigger.

\subsection{Spin preparation for experiments}

In order to prepare the polarized beam for experiments, the beam has to be stored at an energy, where the number of spin rotation between two interaction points is integer or half integer. In case of integer spin rotation between the interaction points, $\gamma G$ is an multiple of six. The stable spin direction is equal at all interaction points. For half integer spin rotation, the stable spin direction alternates from one interaction point to the next. After that, the snake axis has to be adjusted to achieve longitudinal polarization at the interaction points. For a snake axis $^{4}$ of $\phi=-52.1^{\circ}$ or $127.9^{\circ}$ at $\gamma G=192$ $(100.53 \mathrm{GeV})$ the polarization is longitudinal at the interaction points. The resulting stable spin direction in a single snake RHIC ring is presented in Fig. 3. For this setting
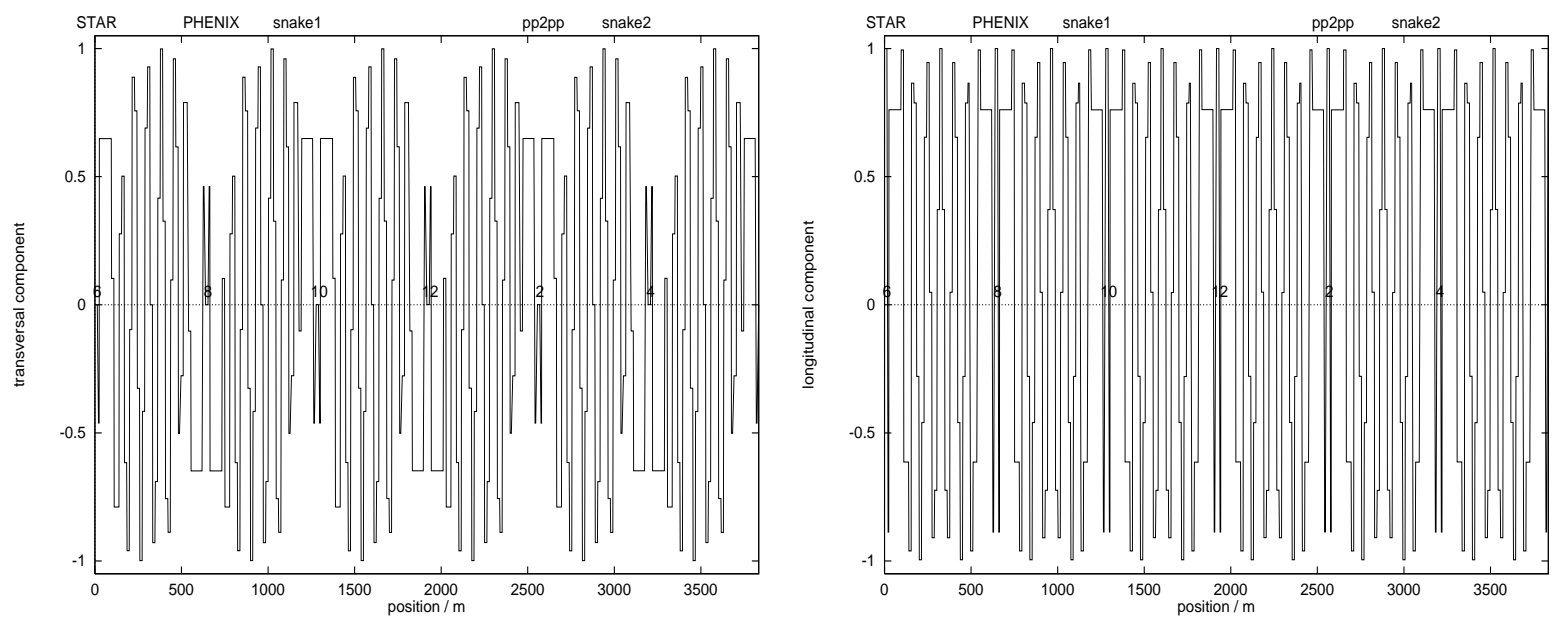

Figure 3: Stable spin direction (left: transversal component; right: longitudinal component) in the blue ring using a single snake with a snake axis of $\phi=-52.1^{\circ}$ or $127.9^{\circ}$ at $\gamma G=192$. The stable spin direction is longitudinal at the interaction points and equal $(-0.6484,0.0000$, $0.7613)$ at the polarimeter. The vertical component of the spin direction is not shown, because it is zero in the whole ring.

the stable spin direction at the polarimeter has an angle of about $-40.4^{\circ}$ with respect to the longitudinal direction. Different energies require different adjustments of the snake axis to get longitudinal polarization at the interaction points. As can be seen in Fig. 4, the angle of the snake axis to get longitudinal polarization at the interaction points is linearly depending on the beam energy. The slope of this curve is $\Delta \phi=4.94^{\circ}$ per $\mathrm{GeV}$. The snake axis is easy to adjust for energies around $100 \mathrm{GeV}$, because the required angle is close to the nominal angle of the snake axis of $\phi=-45^{\circ}$. Due to the dependence of the stable spin direction on the energy, except at the symmetry point of the snake, the momentum spread of the beam leads to a distribution of the spin vectors inside a cone around the stable spin direction. For a momentum deviation of $\Delta p / p=10^{-3}$, the opening angle of this cone is $12^{\circ}$ at the STAR interaction point and $24^{\circ}$ at the PHENIX interaction point. The larger deviation at the PHENIX experiment is caused by the bigger distance

\footnotetext{
${ }^{4}$ The same orientation of the snake axis is required, if any of the other snakes in RHIC is used.
} 


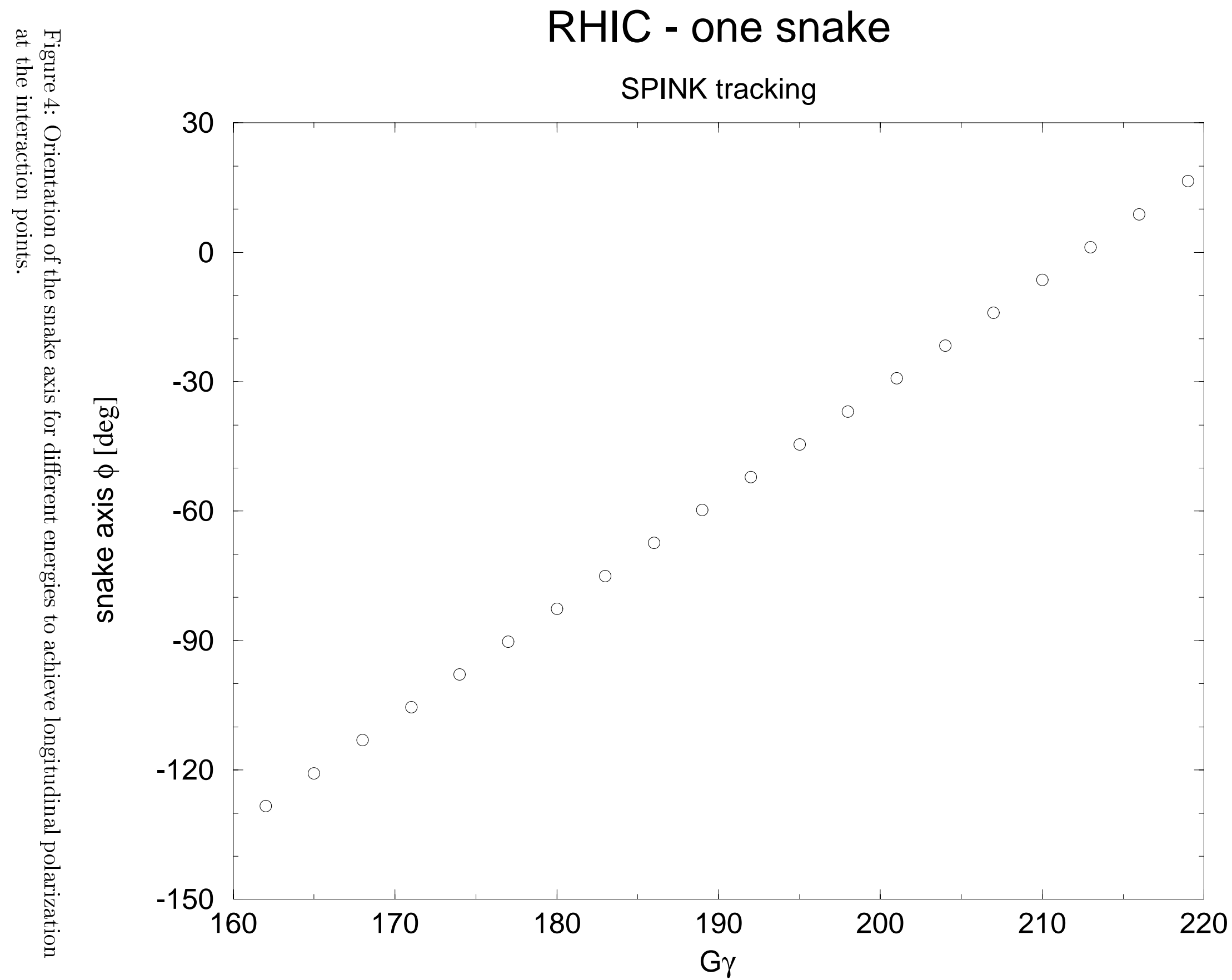


from the symmetry point of the snake. At positions in the ring closer to the symmetry point of the snake, the opening angle of this distribution is getting small. Fig. 5 shows

\section{Polarization with single snake}

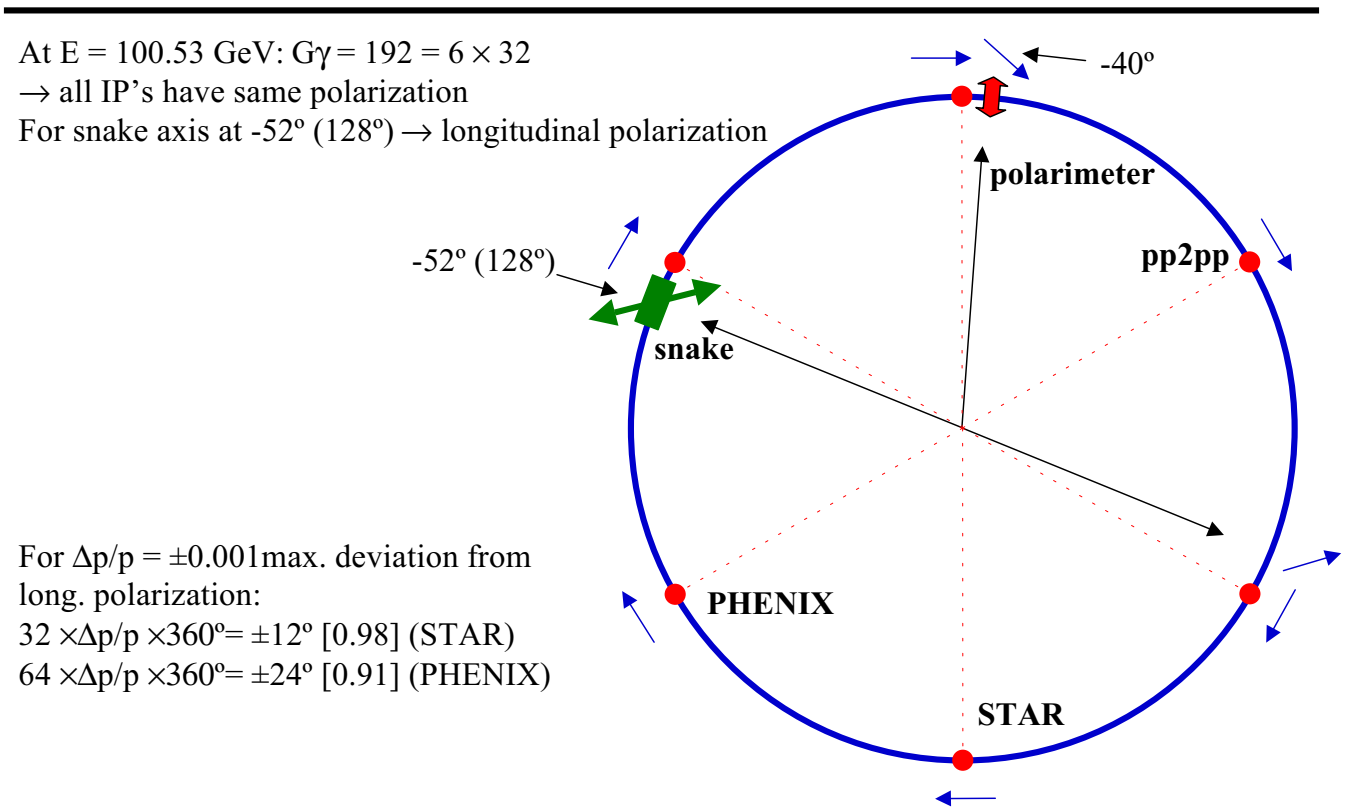

Figure 5: Summary of the snake settings and important properties for providing a longitudinal polarized beam to the experiments at a beam energy of $100.53 \mathrm{GeV}$.

the stable spin direction at the interaction points, the snake, the symmetry point, and the polarimeter for a setting, that could be used to provide longitudinal polarized beam to the experiments at a proposed energy of $100 \mathrm{GeV}$.

\section{Conclusions}

It is possible to accelerate a polarized proton beam with one snake in each RHIC ring above $100 \mathrm{GeV}$ with small polarization losses. For certain energies at a distance of $\Delta E=$ $1.57 \mathrm{GeV}$ from each other, longitudinal polarization at all interaction points in RHIC can be provided. The opening angle of the spin distribution is depending on the distance of the interaction point to the symmetry point of the snake.

\section{References}

[1] Ya.S. Derbenev, A.M. Kondratenko, Part. Accel. Vol.8, 115 (1978).

[2] Design Manual - Polarized Proton Collider at RHIC, Brookhaven National Laboratory (1998), http://www.ags.bnl.gov/rhicspin;

T. Roser, Acceleration of Polarized Protons to High Energies, Proceedings of the Particle Accelerator Conference PAC 99, New York, 26 (1999).

[3] A.U. Luccio et al., Development of the spin tracking program SPINK, Proceedings of the Particle Accelerator Conference PAC 99, New York, 1578 (1999).

[4] H. Grote, F.CH. Iselin, The MAD Program (Methodical Accelerator Design), Version 8.1, User's Reference Manual, CERN/SL/90-13. 